\title{
Algorithmic Study on Position and Movement Method of Badminton Doubles
}

\author{
Yu Xie $\mathbb{D}^{1},{ }^{1}$ Xiaodong Xie $\mathbb{D}^{1},{ }^{1}$ Huan Xia $\mathbb{D D}^{1},{ }^{1}$ and Zhe Zhao ${ }^{2}{ }^{2}$ \\ ${ }^{1}$ Physical Education Institute, Hunan University, Changsha 410082, China \\ ${ }^{2}$ Physical Education Institute, Hunan Normal University, Changsha 410012, China
}

Correspondence should be addressed to Zhe Zhao; 16334@hunnu.edu.cn

Received 27 September 2021; Accepted 16 December 2021; Published 10 January 2022

Academic Editor: Punit Gupta

Copyright ( 2022 Yu Xie et al. This is an open access article distributed under the Creative Commons Attribution License, which permits unrestricted use, distribution, and reproduction in any medium, provided the original work is properly cited.

\begin{abstract}
The algorithms used by schedulers depend on the complexity of the schedule and constraints for each problem. The position and movement of badminton players in badminton doubles competition is one of the key factors to improve the athletes' transition efficiency of offense and defense and the rate of winning matches and to save energy consumption. From the perspective of basic theory, the author conducts research on the position and movement of badminton doubles. Based on the numerical analysis method, the optimal model of standing position and direction composed of 7 nonlinear equations is established. In addition, the final of 10 matches of the super series of the world badminton federation in 2019 was selected as the sample of speed parameters. With the help of MATLAB mathematical analysis software, the numerical model established by the least square method was adopted to optimize the specific standing position and walking model. Ultimately, the optimal solution has been obtained, which can be represented on a plane graph. The optimal position of the attack station should be the blocking area (saddle-shaped area) and the hanging area (circular arc area in the middle). The optimal defensive positioning should be left defensive positioning area (left front triangle area) and right defensive positioning area (right front triangle area), which is consistent with our current experience and research results. The research results use mathematical tools to calculate the accurate optimal position in doubles matches, which has guiding significance to the choice of athletes' position and walking position in actual combat and can also be used as a reference for training, providing a certain theoretical basis for the standing and walking of badminton doubles confrontation. The data collection and operation methods in this study can provide better calculation materials for artificial intelligence optimization and fuzzy operation of motion displacement, which is of great significance in the field of motion, simulation, and the call of parametric functions.
\end{abstract}

\section{Introduction}

As a sport of both recreational fitness and competition, the amount of exercise of badminton can be independently chosen by the participants according to their own conditions, and the requirements for equipment and venues can be high or low. And because the noncontact confrontation reduces the possibility of injury and physical requirements, it has been widely popularized in all parts of the world and has become an Olympic competition [1]. According to the number of participants, the badminton events can be divided into singles, doubles, and three against three. The singles events have high physical requirements [2-4], while the doubles events emphasize more on tactics $[5,6]$, which is essentially an accelerated version of doubles.
In badminton doubles tactics, standing and walking are the basis, which are related to the cooperation between two people and the reasonable distribution of areas [7]. Athletes' technique ability not only needs to have their own characteristics but also must keep strong backcourt tactics ability before, during, and at the same time [8] because, in the game, every detail might affect the competition results; it also makes athletes need to have strong ability of details. The footwork of badminton is an important part of the players' technical and tactical abilities, among which backtracking is the last step of the footwork of badminton, and backtracking connects with the starting link of the next footwork [9]. Reasonable positioning and walking in doubles make the division of labor of teammates clear, makes the preparation of athletes more reasonable, reduces the unnecessary 
running of athletes in the confrontation, saves physical energy, and increases the efficiency of scoring. Quite a few scholars [8-13] have carried out research and discussion on the positions and moves of doubles and reached some consensus. Gawin [10] analyzed the match data and made statistics on what kind of movement mode in doubles is beneficial to gain the advantage in the serve. Zhu qiang et al. [14] used the method of literature review, observation and interview, etc., to discuss the position of attack and defense switch in badminton doubles. Lin [15] also used similar research methods to analyze and discuss Yang wang Xiao li's position in 10 doubles matches from 2013 to 2014.

It can be seen that, in addition to forming some empirical consensus, there have also been some relevant studies on the positioning and movement of doubles. Partial least squares correlation analysis (PLSCA) (Abdi and Williams, 2013; Weaving et al., 2019) [16] was used to investigate the composite relationship between perceived wellness status and technical-tactical performance for both the forwards' and backs' positional groups as per previous methods [17] (Emmonds et al., 2020). However, the conclusions of these studies are all qualitative and empirically based, which may be practical but not rigorous. In order to scientifically optimize the positioning of doubles in training and actual combat, it is very important to model and analyze the positioning of doubles, theoretically explore the most reasonable positioning and movement of doubles, and avoid the existing errors. Therefore, based on the mathematical model [16-19], the following research is carried out on the optimization of badminton doubles' position and movement, in order to provide some theoretical basis on this issue.

\section{The Positioning Model of Doubles Based on the Analytic Method}

2.1. The Basic Idea of Model Building. In a badminton match, when a player of his own side hits the ball, the time it takes for the ball to fly from his own field to the field of his opponent is the time for the player of his own side to stand. Similarly, after the opponent hits the ball, the time for the ball to fly from his court to his opponent's court is the time for his opponent to start and hit the ball. This is the basic rhythm of badminton.

As can be seen from Figure 1, the time required for the ball to fly from one side of the field to different areas of the opposite side of the field is different, that is, the time from starting to hitting is different for the incoming ball from different landing points. For example, the split lob is faster and shorter than the high ball, so the player who wants to catch the ball should start and run to the right hitting area in a shorter time. Of course, there is a certain difference between the running speed of the players' forward net footwork and the running speed of the players' backward retreats' footwork. To sum up, ignoring the secondary influencing factors, the players should have a corresponding optimal positioning point for the position of the ball when facing the opponent's shot. From this positioning point, it should be equally difficult for them to return several furthest corners of the area they are responsible for. In order to get this position, the emphasis is on the evaluation of the difficulty of connecting each ball at the farthest corner. As the ball travels from the opposing field to each of these farthest corners, it corresponds to the distance the player must run and the time he has to start to hit the ball, which is the amount of speed the player must put into catching the ball. The magnitude of this required speed is a measure of difficulty. So, the optimal positioning should allow the player at that point to run at the two farthest corners of the net at the same speed. The two farthest corners of the baseline should run at the same speed. Moreover, the running speed towards the furthest corner of the net and the furthest corner of the baseline should satisfy the proportional relation between the running speed of the net footwork and the running speed of the back-and-back footwork.

2.2. Badminton Court Coordinate System Creation. In order to calculate the distance better, an optimization model is established based on the analytical method in this paper. Therefore, the analytical coordinate system of the site should be built first to obtain the coordinate values of each key point. One end of the center line of the net is taken as the origin of the coordinate system. The $X$-axis is along the long side of the court, while the $Y$-axis bisects the opposite side of the court along the direction of the net, as shown in Figure 2.

In Figure 2, the $X$-axis represents the longitudinal distance in the direction of the badminton court sideline, the $Y$ axis represents the transverse distance in the direction of the center line, and the intersection of one side sideline and the middle is the origin. Doubles position is the end of the movement to the position, the starting point to hit the ball, and the basis of other doubles tactics. Therefore, each position corresponds to the position of the ball when an opponent hits the ball. In order to better illustrate the optimization process, the ball is located at the corner of the opponent's baseline (G spot in Figure 2) as an example; see Figure 2, for the coordinates of the two players' position points (points $J$ and $\mathrm{K}$ on Figure 2) and the farthest corner points (points A, B, C, D, E, and F on Figure 2) in the area they are responsible for (CD is the dividing line of the area). Considering that when the opponent hits the ball from the baseline and ignores the situation that the ball rolls over the net with a small probability, the ball should not fall into the area surrounded by the two sideline of the line AE, the field, and the net line ( $Y$-axis), so there is no distribution of points needing to catch the ball in this area. At this point, there are seven unknown coordinate parameters, including the twoaxis coordinates $x 1$ and $y 1$ at point J, the two-axis coordinates $x 2$ and $y 2$ at point $\mathrm{K}$, the two-axis coordinates $m$ and $n$ at point $\mathrm{C}$, and the $y$-coordinate $p$ at point $\mathrm{D}$, where the coordinates $x 1$ and $y 1$ and $x 2$ and $y 2$ of $J$ are exactly the coordinate values to be obtained.

2.3. Optimization Model Creation. As shown in Figure 2, the ball falls from point $G$ to $A, B$, or C. Points B, D, and F correspond to the point where the ball clicks back from $\mathrm{G}$. The horizontal component of the average flying speed of the 


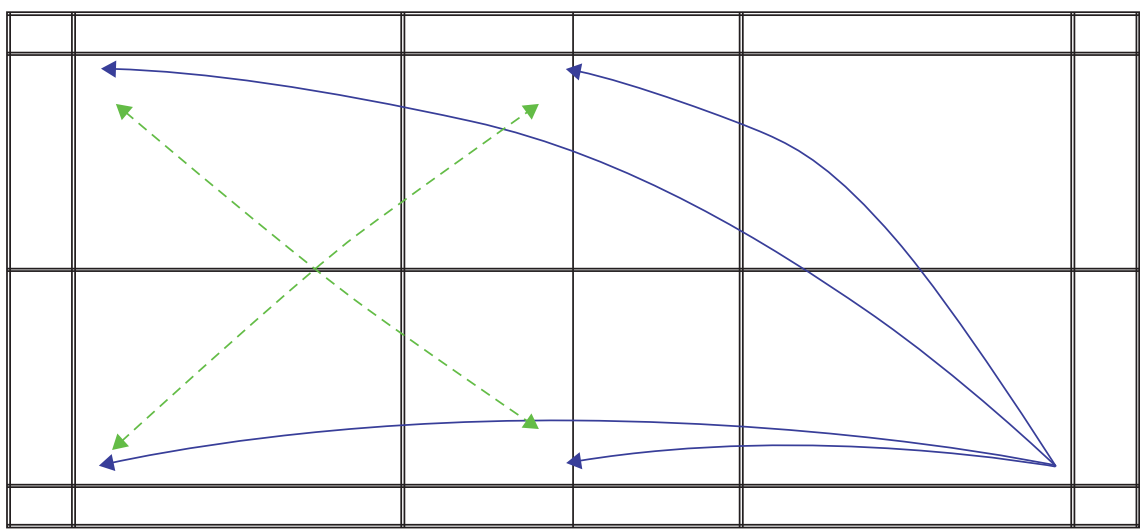

4 Badminton flight path

$4--$ Run the line

FIgURE 1: Badminton flight and running circuit diagram.

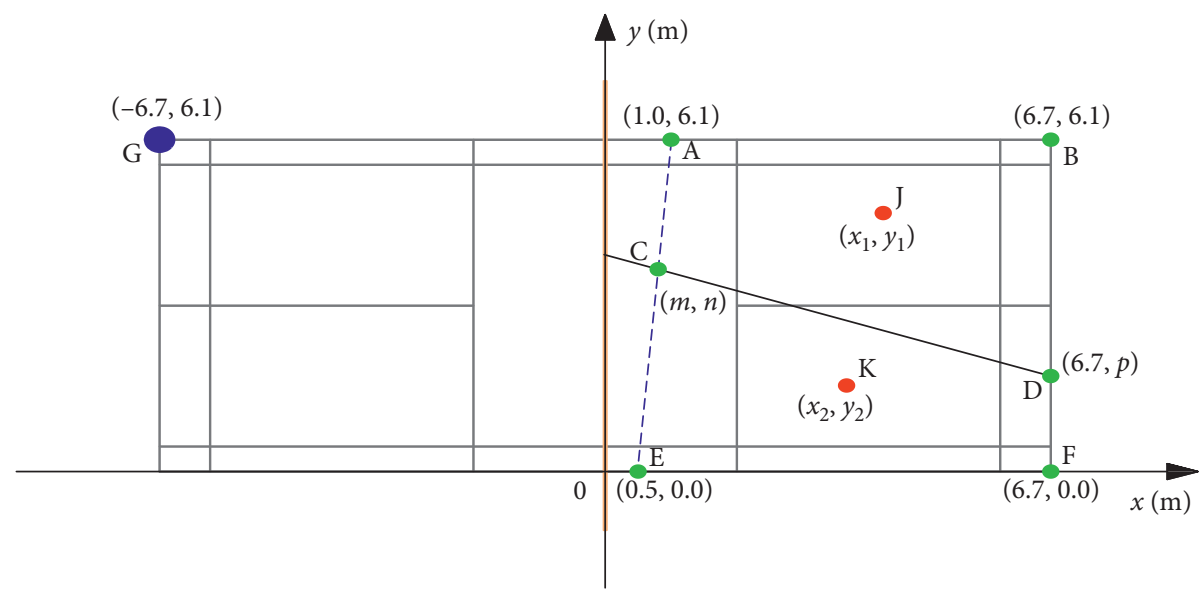

Figure 2: Analytical coordinate system of badminton doubles court and station.

ball when hitting the ball is $\mathrm{m} / \mathrm{s}$. The average speed of returning the ball after receiving the ball is $\mathrm{m} / \mathrm{s}$. According to the physical relationship between flight distance, flight speed, and flight time, the time (unit: $s$ ) of the ball from point $\mathrm{G}$ to points $\mathrm{A}, \mathrm{B}, \mathrm{C}, \mathrm{D}, \mathrm{E}$ and $\mathrm{F}$ is

$$
\begin{aligned}
& t_{\mathrm{GA}}=7.7 / \overline{V_{\mathrm{ds}}}, \\
& t_{\mathrm{GB}}=13.4 / \overline{V_{\mathrm{hc}}}, \\
& t_{\mathrm{GC}}=\frac{\sqrt{(6.7+m)^{2}+(6.1-n)^{2}}}{\overline{V_{\mathrm{ds}}}}, \\
& t_{\mathrm{GD}}=\frac{\sqrt{13.4^{2}+(6.1-p)^{2}}}{\overline{V_{\mathrm{hc}}}}, \\
& t_{\mathrm{GE}}=\frac{\sqrt{7.2^{2}+6.1^{2}}}{\overline{V_{\mathrm{ds}}}}=\frac{9.4}{\overline{V_{\mathrm{ds}}}},
\end{aligned}
$$

$$
e w t_{\mathrm{GF}}=\frac{\sqrt{13.4^{2}+6.1^{2}}}{\overline{V_{\mathrm{hc}}}}=\frac{14.7}{\overline{V_{\mathrm{hc}}}} .
$$

Assuming that the opponent hits the ball at the exact time when the receiver starts, then according to the physical relationship between running distance, running speed, and running time, the running speed required by the player at point $\mathrm{J}$ to catch the ball at $\mathrm{A}, \mathrm{B}, \mathrm{C}$, and $\mathrm{D}$ is

$$
\begin{aligned}
& V_{\mathrm{JA}}^{n}=\frac{\sqrt{\left(x_{1}-1.0\right)^{2}+\left(6.1-y_{1}\right)^{2}}}{t_{\mathrm{GA}}}, \\
& V_{\mathrm{JB}}^{b}=\frac{\sqrt{\left(6.7-x_{1}\right)^{2}+\left(6.1-y_{1}\right)^{2}}}{t_{\mathrm{GB}}}, \\
& V_{\mathrm{JC}}^{n}=\frac{\sqrt{\left(x_{1}-m\right)^{2}+\left(y_{1}-n\right)^{2}}}{t_{\mathrm{GC}}},
\end{aligned}
$$




$$
V_{\mathrm{JD}}^{b}=\frac{\sqrt{\left(6.7-x_{1}\right)^{2}+\left(y_{1}-p\right)^{2}}}{t_{\mathrm{GD}}} .
$$

The running speeds required by players at point $\mathrm{K}$ to catch the ball at four points $\mathrm{C}, \mathrm{D}, \mathrm{E}$, and $\mathrm{F}$ are

$$
\begin{aligned}
& V_{\mathrm{KC}}^{n}=\frac{\sqrt{\left(x_{2}-m\right)^{2}+\left(n-y_{2}\right)^{2}}}{t_{\mathrm{GC}}}, \\
& V_{\mathrm{KD}}^{b}=\frac{\sqrt{\left(6.7-x_{2}\right)^{2}+\left(p-y_{2}\right)^{2}}}{t_{\mathrm{GD}}}, \\
& V_{\mathrm{KE}}^{n}=\frac{\sqrt{\left(x_{2}-0.5\right)^{2}+y_{2}^{2}}}{t_{\mathrm{GE}}}, \\
& V_{\mathrm{KF}}^{b}=\frac{\sqrt{\left(6.7-x_{2}\right)^{2}+y_{2}^{2}}}{t_{\mathrm{GF}}} .
\end{aligned}
$$

In order to simplify the calculation, at the same time, according to the split in the following section hanging ball when the average speed of horizontal component of the ball when the ball hits the back of the level of the average speed component, athletes do back to the bottom line of footwork when running with speed and athletes to the net do online gait as running speed of the related research (specific data discussed in Section 3), the ratio of discovery can be approximately considered as the constant value, which is

$$
\begin{gathered}
\frac{\overline{V_{\mathrm{hc}}}}{\overline{V_{\mathrm{ds}}}}=K_{1}, \\
\frac{V^{n}}{V^{b}}=K_{2} .
\end{gathered}
$$

According to the basic idea in Section 2.1, when the athletes at point $\mathrm{J}$ and at point $\mathrm{K}$ meet the requirements of equation (16), the speed required to catch the ball at the farthest corner point should be in the optimal position:

$$
\begin{gathered}
V_{\mathrm{JA}}^{n}=K_{2} V_{\mathrm{JB}}^{b}=V_{\mathrm{JC}}^{n}=K_{2} V_{\mathrm{JD}}^{b}, \\
V_{\mathrm{KC}}^{n}=K_{2} V_{\mathrm{KD}}^{b}=V_{\mathrm{KE}}^{n}=K_{2} V_{\mathrm{KF}}^{b} .
\end{gathered}
$$

By substituting equations (1) (15) into equations (17) and (18), we can obtain

$$
\begin{aligned}
13.4 \sqrt{\left(x_{1}-1.0\right)^{2}+\left(6.1-y_{1}\right)^{2}} & =7.7 K_{1} K_{2} \sqrt{\left(6.7-x_{1}\right)^{2}+\left(6.1-y_{1}\right)^{2}}, \\
\sqrt{\left(x_{1}-1.0\right)^{2}+\left(6.1-y_{1}\right)^{2}} \cdot \sqrt{(6.7+m)^{2}+(6.1-n)^{2}} & =7.7 \sqrt{\left(x_{1}-m\right)^{2}+\left(y_{1}-n\right)^{2}}, \\
\sqrt{\left(x_{1}-1.0\right)^{2}+\left(6.1-y_{1}\right)^{2}} \cdot \sqrt{13.4^{2}+(6.1-p)^{2}} & =7.7 K_{1} K_{2} \sqrt{\left(6.7-x_{1}\right)^{2}+\left(y_{1}-p\right)^{2}}, \\
9.4 K_{1} K_{2} \sqrt{\left(6.7-x_{2}\right)^{2}+\left(p-y_{2}\right)^{2}} & =\sqrt{\left(x_{2}-0.5\right)^{2}+y_{2}^{2}} \cdot \sqrt{13.4^{2}+(6.1-p)^{2}}, \\
9.4 \sqrt{\left(x_{2}-m\right)^{2}+\left(n-y_{2}\right)^{2}} & =\sqrt{\left(x_{2}-0.5\right)^{2}+y_{2}^{2}} \cdot \sqrt{(6.7+m)^{2}+(6.1-n)^{2}}, \\
14.7 \sqrt{\left(x_{2}-0.5\right)^{2}+y_{2}^{2}} & =9.4 K_{1} K_{2} \sqrt{\left(6.7-x_{2}\right)^{2}+y_{2}^{2}} .
\end{aligned}
$$

Since point $\mathrm{C}$ is located on the point line $\mathrm{AE}$, it should be satisfied:

$$
n=12.2 m-6.1 \text {. }
$$

\section{Model Parameters and Solutions}

3.1. Model Parameters: Speed Parameters. In order to simplify and solve the established optimization model, this paper tests the horizontal component of the average flying speed of the ball when splitting the crane, the horizontal component of the average flying speed of the ball when hitting the backcourt ball, the running speed when the player makes a backward step to the In this paper, and the final videos of 10 matches of the world badminton federation super series in the recent year were selected as the statistical space to conduct sample statistics of various speed parameters. The source of the matches is shown in Table 1. A total of 668 effective sum ratio data and 382 effective sum ratio data were obtained, and their distribution is shown in Figure 3.

Through fitting, it can be approximated to a fixed value of 0.7657 and a fixed value of 1.3026. In equations (19) (25) of the optimization model, it is always multiplied, so it can be regarded as a parameter.

3.2. Model Solution. Models (19)-(25) are for nonlinear equations, and the equation number equals the number of variables (which can be regarded as known quantity), so a 
TABLE 1: Model data source statistics table.

\begin{tabular}{|c|c|c|c|}
\hline Event name & Category & Contestant & $\begin{array}{l}\text { The number of } \\
\text { data }\end{array}$ \\
\hline Yubo Cup Final 2018 & $\begin{array}{l}\text { Women's } \\
\text { doubles }\end{array}$ & Yuki Fukushima Katsuka Hiroda vs. Zonko pan Ravinda & 69 \\
\hline 2018 World Championships & Men's doubles & Li Junhui/Liu Yuchen vs. Jiamura Kenshi/Yueda Qigu & 77 \\
\hline $\begin{array}{l}2018 \text { World Championship } \\
\text { Final }\end{array}$ & $\begin{array}{l}\text { Women's } \\
\text { doubles }\end{array}$ & $\begin{array}{l}\text { Yoshiyuki Fukushima/Hiroda Katsuhwa vs. Maayu Matsumoto } \\
\text { Naga }\end{array}$ & 66 \\
\hline 2018 World Badminton Final & $\begin{array}{l}\text { Women's } \\
\text { doubles }\end{array}$ & Takahashi Lihua/Matsuomi Sasaki vs. Li Shaoxi/Shen Shengzan & 65 \\
\hline 2019 Sudiman Cup Final & Men's doubles & Li Junhui/Liu Yuchen vs. Watanabe Yuda/Endo Dayou & 72 \\
\hline 2018 World Badminton Final & $\begin{array}{l}\text { Women's } \\
\text { doubles }\end{array}$ & Takahashi Lihua/Matsuomi Sasaki vs. Li Shaoxi/Shen Shengzan & 65 \\
\hline $\begin{array}{l}2019 \text { World Championship } \\
\text { Final }\end{array}$ & Men's doubles & Setiawan/Ahsan vs. Tomoki Zhuo/Kobayashi & 59 \\
\hline $\begin{array}{l}2018 \text { World Championship } \\
\text { Final }\end{array}$ & $\begin{array}{l}\text { Women's } \\
\text { doubles }\end{array}$ & $\begin{array}{l}\text { Yoshiyuki Fukushima/Hiroda Katsuhwa vs. Maayu Matsumoto/ } \\
\text { Naga }\end{array}$ & 66 \\
\hline 2019 World & $\begin{array}{l}\text { Women's } \\
\text { doubles }\end{array}$ & $\begin{array}{c}\text { Badminton final Mayou Matsumoto/Nagawara vs. chen Morin/Jia } \\
\text { Yifan }\end{array}$ & 64 \\
\hline $\begin{array}{l}2019 \text { World Championship } \\
\text { Final }\end{array}$ & $\begin{array}{l}\text { Women's } \\
\text { doubles }\end{array}$ & Mayou Matsumoto/Nagawara/Yuki Fukushima/Kohwa Hiroda & 71 \\
\hline
\end{tabular}

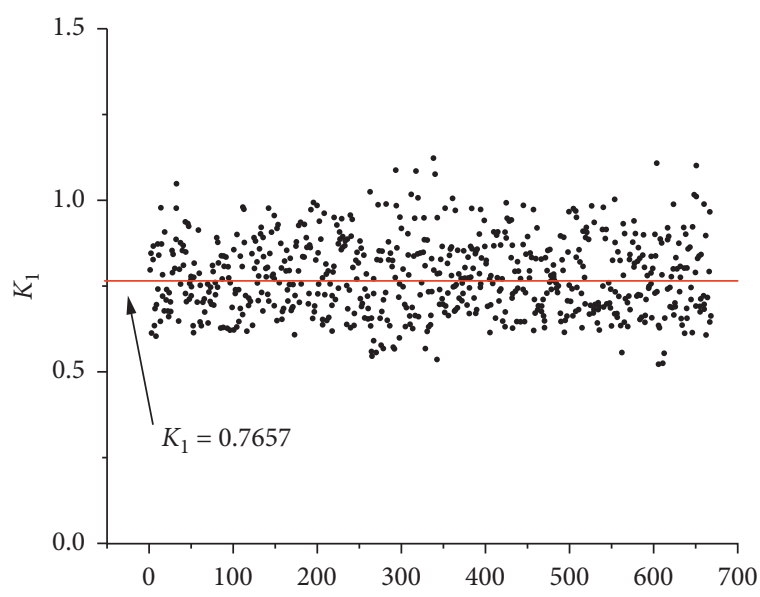

(a)

FIGURE 3: Velocity parameter distribution diagram.

given interval may have a solution. Now, the fsolve function of MATLAB mathematical software is used for trial calculation and solution. According to the help file of MATLAB, the fsolve function is to use the least square method to solve the nonlinear equations, and the general solution formula is

$$
X=\text { FSOLVE(FUN, X0, OPTIONS), }
$$

Where FUN is the nonlinear system of equations requiring solution. For this paper, equations (19) (25) are the nonlinear system and the initial value of the variable. Because the solution process has a certain dependence on the numerical value, in this paper, the understanding range is roughly calculated according to the experience. Then, we set the initial value. OPTIONS represents the structure created for OPTIMSET, which is the default value for this optimization parameter.

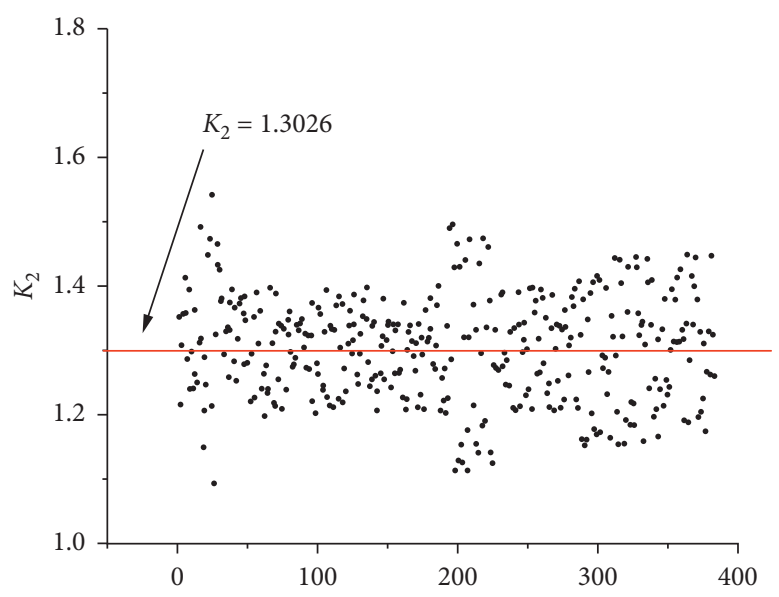

(b)

(a) $K_{1}$ distribution diagram. (b) $K_{2}$ distribution diagram.

\begin{tabular}{cccccccc}
\hline & $x_{1}$ & $y_{1}$ & $x_{2}$ & $y_{2}$ & $m$ & $n$ & $p$ \\
\hline Results & 2.8661 & 4.6844 & 2.6353 & 1.9088 & 0.7839 & 3.4636 & 2.9784 \\
\hline
\end{tabular}

In the input equations (19) (25) and the estimated initial values of each parameter, the number of cycles is set to 1000 generations. The final results of each parameter are shown in Table 2. The various errors corresponding to the solutions obtained through numerical iteration are shown in Table 3.

Put the result back into the field map, and Figure 4(a) can be obtained. The point of the left field is the position of the ball when the opponent hits the ball. The point on the right side of the field is the optimal station point of the two players of the team based on the optimization of analytical method. By the same method, the optimal solution of the position required by 
TABLE 3: Errors of equations (19) (25) corresponding to solutions.

\begin{tabular}{|c|c|c|c|c|c|c|c|}
\hline Equation & (19) & (20) & (21) & (22) & (23) & (24) & (25) \\
\hline Error & $-8.74 E-07$ & $-1.18 E-07$ & $-9.76 E-07$ & $8.91 E-07$ & $-1.37 E-07$ & $-9.30 E-07$ & $8.26 E-14$ \\
\hline
\end{tabular}

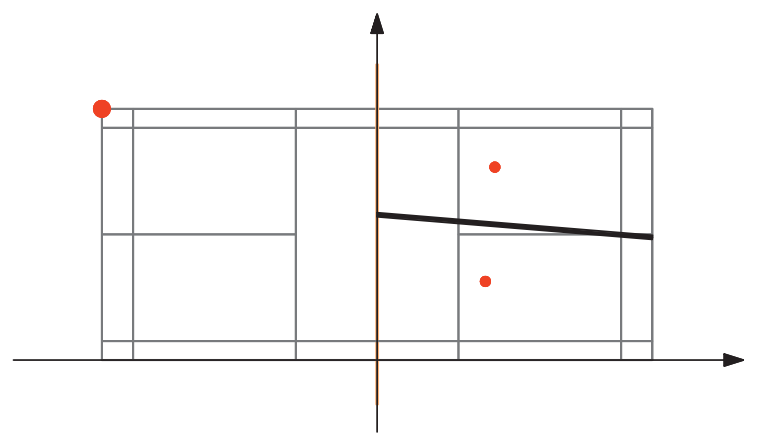

(a)

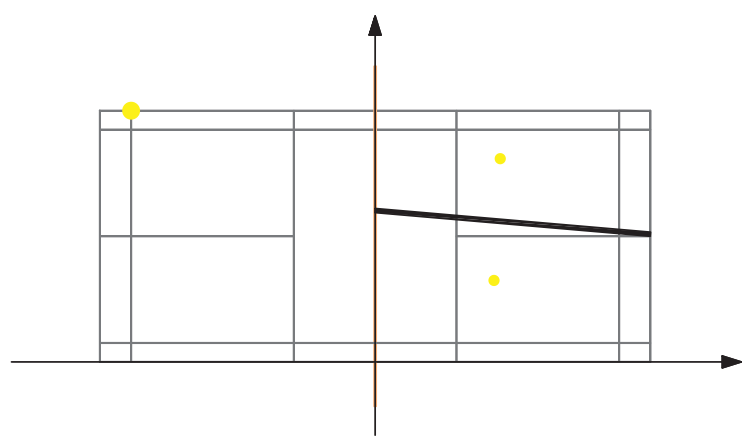

(c)

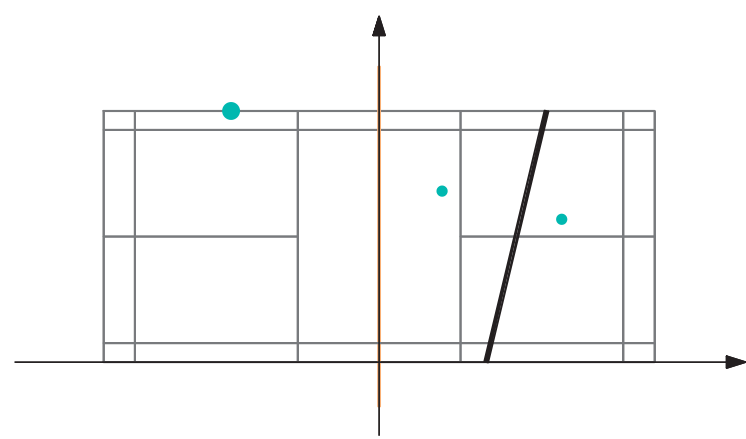

(e)

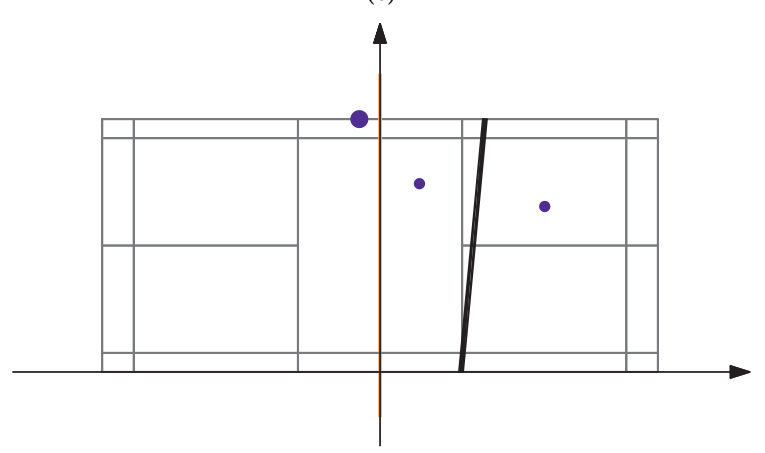

(g)

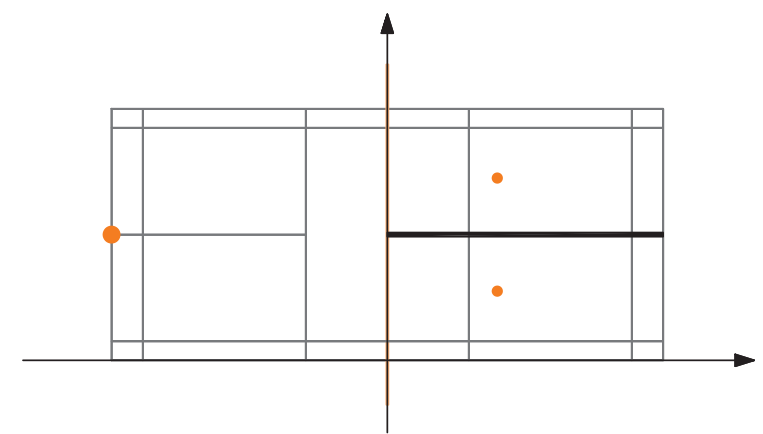

(b)

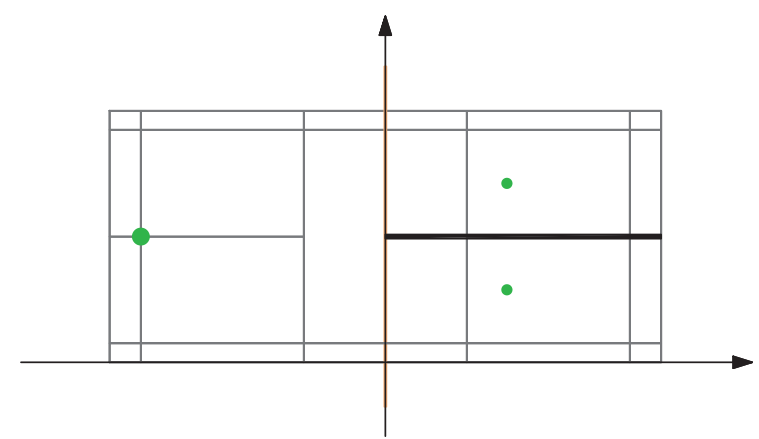

(d)

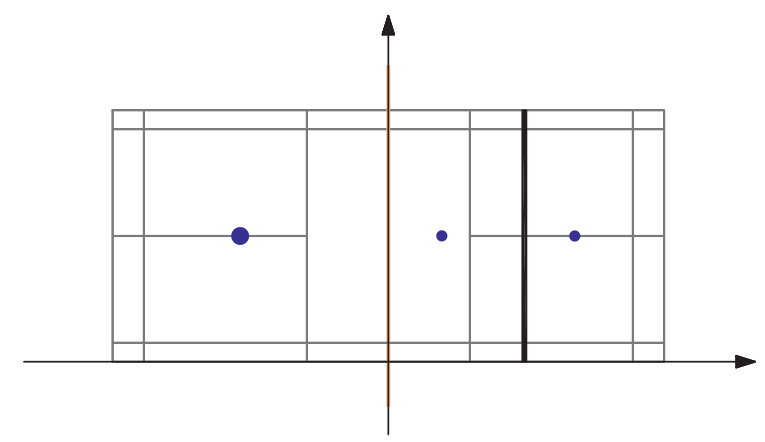

(f)

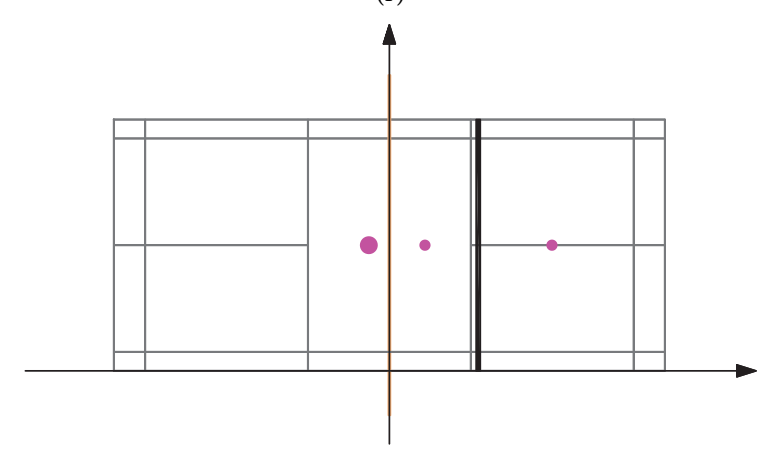

(h)

Figure 4: Results of optimal positioning when the ball is at each key point in the opposite side field area. (a) Scenario 1. (b) Scenario 2. (c) Scenario 3. (d) Scenario 4. (e) Scenario 5. (f) Scenario 6. (g) Scenario 7. (h) Scenario 8. 


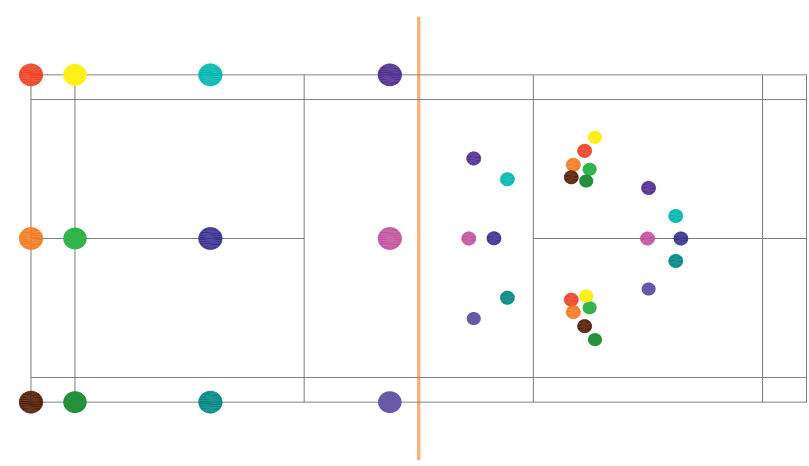

FIGURE 5: Summary of optimized station positioning results.
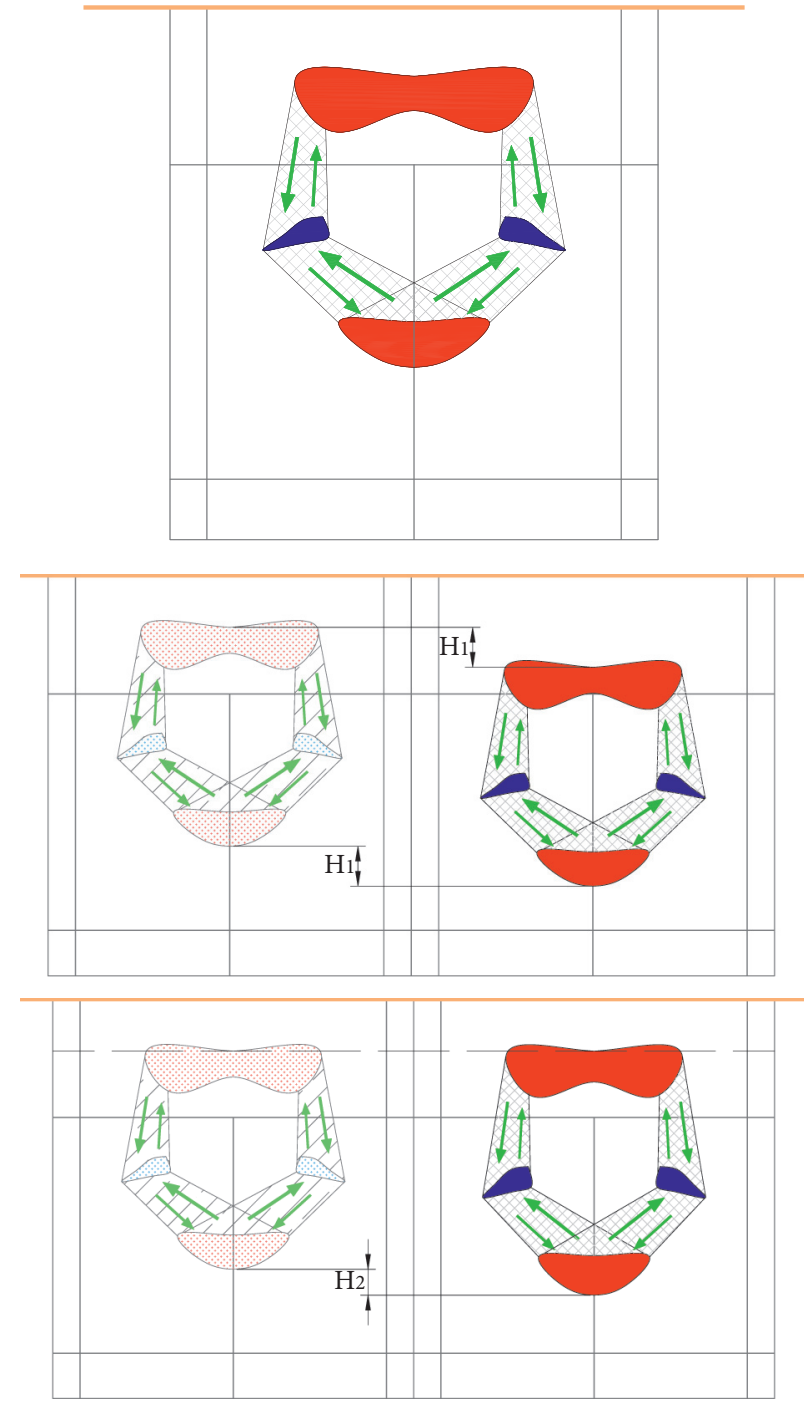

FIgURE 6: Station area and rotation path.

the opponent when the ball is at the other key points of the opponent's court can be calculated, as shown in Figures 4(b) 4(h). All the obtained optimized station positions and the results obtained according to the symmetry relation are collected in the same figure, as shown in Figure 5. The points in the left and right field areas of the same color correspond to the position of the ball and the corresponding relationship between the positions of the square and the station. 


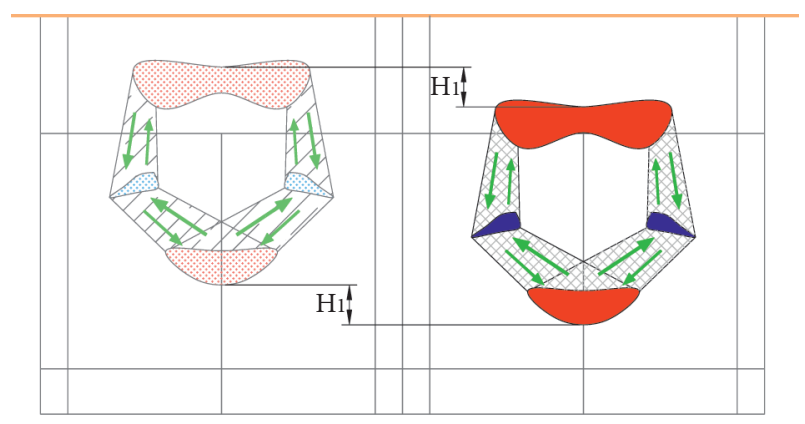

(a) Backward translation

(b) Longitudinal lengthening

Figure 7: Conjecture of running circle results when more factors are considered. (a) Backward translation. (b) Longitudinal lengthening.

\section{Analysis and Discussion}

In Figures 4 and 5, the $X$-axis represents the longitudinal distance in the direction of the badminton court sideline, the $Y$-axis represents the transverse distance in the direction of the center line, and the intersection of one side sideline and the middle is the origin. The different color dots sign the footfall dots and the directions. Three spline curves were used to connect the stations of each side in the center and right field in Figure 4 to obtain two offensive areas (red area) and two defensive areas (blue area), as shown in Figure 6.

There are two offensive areas; one is in the front of the field, in the actual combat process, which should be responsible for the front of the net, so it can be called the net position area; the other is in the back court, in the actual combat process, which should be responsible for the back court attack, so it can be called the killing and hanging station area. The two defensive areas are located in the left and right field, respectively, which can be called the left guard area and the right guard area (in real combat rotation, players should try their best to move in a straight line to reduce the distance of moving, so they should use a straight line to connect the boundaries of the offensive area and the defensive area). The rotation path in Figure 6 can be obtained. In the actual combat process, the so-called offensive to defensive and defensive to offensive are the two players who continue to choose a reasonable position between the offensive zone and the defensive zone through these paths. Based on the analytical method, we obtain Figure 6, which is obtained by the optimization study of such a stance area and rotary path graph; you can connect the attack area and defensive area and their rotary path as a special "movement." Using the running circle, we can guide the positioning and walking training of doubles players and even put the running circle on the field, so as to develop the fixed thinking and concept of positioning and field of doubles players. In this way, the possible errors of positioning or moving can be reduced due to panic in high-speed doubles competition.

This study is based on the mathematical analysis method to optimize the position of doubles, and a running circle is preliminary calculated. For example, consider that, in practice, when players hit the ball at the baseline, they often choose to kill the ball vigorously instead of hitting it to the far corner of their own side. When the player receives the ball in front of the court, he can use a certain arm span and racket length; the center of gravity does not move to the position of the ball. It also takes time for the athlete to start the turn from the position. Taking the secondary influencing factors into account will be the next step for more deepening the research on the optimization of the target on the basis of the overall train of thought. It is assumed that when the above factors are taken into account, the resulting running circle may be the result of a backward shift (as shown in Figure $7(\mathrm{a})$ ) or a longitudinal stretch (as shown in Figure 7(b)) compared with the current one. These assumptions need to be further studied to confirm whether they are reasonable. This kind of data has the position characteristics of relatively optimized fuzzy algorithm. These features can provide reference for data collection and feature analysis of subsequent sports competitions of multiple athletes. After collecting the location data of multiple multievent competitions, the database can optimize the multipoint fuzzy computing of computer cloud, so as to obtain a better model.

\section{Conclusion}

Based on the analytical method, this paper establishes the optimal model of badminton doubles position, obtains the relevant speed parameters through statistical analysis, then solves the model by numerical method, and draws the following conclusions.

(1) Based on the analytical method, an optimization model is established. By inputting reasonable speedrelated parameters, the specific points of the optimal positions of the two players in badminton doubles can be solved.

(2) The specific distribution of these optimal stations constitutes four regions, namely, the area of net closing (the saddle-shaped area in the front), the area of killing and hanging (the circular arc area in the middle), the area of left guard (the triangle area in the left front), and the area of right guard (the triangle area in the right front).

(3) The rotation path can be obtained by connecting the boundary between the offensive zone and the defensive zone. The two players can constantly choose a reasonable position between the offensive zone and the defensive zone through the rotation path to realize the switch from offense to defense and from defense to offense.

(4) The offensive zone and the rotation paths that connect them combine to form a running circle, which can be used to guide doubles players in positioning training.

(5) As a model of multiplayer sports, this study can provide more detailed data and statistics. The research also provides a reference model for the collection and calculation of multievent and multiproject secretaries of subsequent cloud 
computing, to obtain the optimization of algorithm and model.

\section{Data Availability}

The datasets used and/or analyzed during the current study are available from the corresponding author upon reasonable request.

\section{Conflicts of Interest}

The authors declare that there are no conflicts of interest with respect to the research, authorship, and/or publication of this article.

\section{References}

[1] J. Y. Guillain, Badminton: An Illustrated History-From Ancient Pastime to Olympic Sport, Editions Publibook, France, 2012.

[2] M. K. Chin, A. S. Wong, R. C. So, O. T. Siu, K. Steininger, and D. T. Lo, "Sport specific fitness testing of elite badminton players," British Journal of Sports Medicine, vol. 29, no. 3, pp. 153-157, 1995.

[3] P. Majumdar, G. L. Khanna, V. Malik, S. Sachdeva, M. Arif, and M. Mandal, "Physiological analysis to quantify training load in badminton," British Journal of Sports Medicine, vol. 31, no. 4, pp. 342-345, 1997.

[4] O. Faude, T. Meyer, F. Rosenberger, M. Fries, G. Huber, and W. Kindermann, "Physiological characteristics of badminton match play," European Journal of Applied Physiology, vol. 100, no. 4, pp. 479-485, 2007.

[5] D. Cabello Manrique and J. J. Gonzalez-Badillo, "Analysis of the characteristics of competitive badminton," British Journal of Sports Medicine, vol. 37, no. 1, pp. 62-66, 2003.

[6] M. Blomqvist, P. Luhtanen, and L. Laakso, "Comparison of two types of instruction in badminton," European Journal of Physical Education, vol. 6, no. 2, pp. 139-155, 2001.

[7] M. Blomqvist, Game Understanding and Game Performance in Badminton: Development and Validation of Assessment Instruments and Their Application to Games Teaching and Coaching, University of Jyväskylä, Jyväskylä, Finland, 2001.

[8] H. Abdi and L. J. Williams, "Partial least squares methods: partial least squares correlation and partial least square regression," Methods in Molecular Biology, vol. 27, pp. 549-579, 2013.

[9] D. Bates, M. Mächler, B. Bolker, and S. Walker, "Fitting linear mixed-effects models using lme4," Journal of Statistical Software, vol. 67, no. 1, 2015.

[10] W. Gawin, C. Beyer, H. Hasse, and D. Büsch, "How to attack the service: an empirical contribution to rally opening in world-class badminton doubles," International Journal of Performance Analysis in Sport, vol. 13, no. 3, pp. 860-871, 2013.

[11] D. Jin-Biao, "Analysis on the application of technique and tactics in badminton men's doubles," Journal of Shanghai institute of sport, vol. 22, no. 2, pp. 36-40, 1998.

[12] li Cheng, Comparative Analysis on Technique and Tactics of Mixed Doubles Badminton between China and Foreign Countries, Chengdu Institute of Physical Education, China, 2013.

[13] T. Zhao, Analysis on the Technical and Tactical Characteristics of the World's Outstanding Badminton Men's Doubles Players, Beijing Sport University, Beijing, China, 2010.
[14] Q. Zhu and Y. Tang, "On the skills of defense and attack conversion in badminton doubles," Contemporary sports science and technology, vol. 4, no. 16, pp. 41-42, 2014.

[15] Q. Lin, Analysis and Research on Coordination Position in Women's Badminton Doubles, Shandong institute of physical education, Shandong, China, 2016.

[16] R. Kager, Q. W. Ma, and J. L. Wang, Optimality Theory, Cambridge University Press, Cambridge, United Kingdom, 1999.

[17] J. J. McCarthy, A Thematic Guide to Optimality Theory, Cambridge University Press, Cambridge, United Kingdom, 2002.

[18] B. Tesar, Learnability in Optimality Theory, Mit Press, Cambridge, Massachusetts, United States, 2000.

[19] D. Archangeli, Optimality Theory, John Wiley \& Sons, New York, NY, USA, 2006. 